\title{
Analyse du matériel pédagogique en matière de ponctuation dans l'école primaire algérienne
}

\author{
Hassiba Addou \\ Faculté des lettres, des langues et des arts, Université Djillali Liabès, Sidi-Bel-Abbès-Algérie
}

Article reçu le 2 Octobre 2015, accepté 24 janvier 2016, version finale 27 janvier 2016

http://dx.doi.org/10.5565/rev/jtl3.650

\section{Résumé}

Savoir écrire, lire et communiquer nécessite un apprentissage particulier. L'acquisition des compétences démarcatives dans l'enseignement/apprentissage du français langue étrangère, suppose un usage à bon escient de la ponctuation. A l'école primaire algérienne, ce genre d'apprentissage reste sensible puisqu'il dépend, essentiellement, des programmes et de l'âge des apprenants. C'est dans cette optique que nous développerons une analyse $d u$ matériel pédagogique afin d'examiner l'adéquation entre la conception et l'élaboration de l'objet à enseigner la ponctuation, et les composantes des compétences visées, notamment dans les manuels scolaires et les cahiers d'activités.

Mots-clés : ponctuation, apprentissage, compétence, programme primaire, manuel scolaire

\begin{abstract}
Knowing how to write, read and communicate requires special training. The acquisition of demarcation skills in French as a Foreign Language implies a good understanding and ability to use punctuation. At primary schools in Algiers, the success of this genre of learning largely depends on the type of learning programs that are implemented as well as the age of the target students. Along these lines, this article provides an analysis of pedagogical materials aimed to examine the adequacy of the design and development of the artifacts used to teach punctuation, and related key target competences, particularly in textbooks and activities books.
\end{abstract}

Keywords: punctuation, learning, competency, primary program, textbooks

\section{Resumen}

Saber escribir, leer y comunicar necesita una formación muy particular. La adquisición de las competencias demarcadoras en el aprendizaje de francés como lengua extranjera supone una comprensión de la puntuación. En la escuela primaria argelina, el éxito de este genero de aprendizaje depende esencialmente del tipo de programa y la edad del alumnado. Desde este punto de vista, el artículo describe un análisis del material pedagógico con el fin de examinar la adecuación entre el diseño (su concepción) y la elaboración de los objeto utilizado para que enseñar la puntuación, y los componentes relacionados a las competencias 
aludidas, particularmente en los manuales escolares y los cuadernos de actividades complementarias.

Palabras clave: Puntuación, aprendizaje, competencia, programa escolar primario, libros de textos

\section{Cadre de l'étude}

L'usage des signes de ponctuation dans l'acquisition de l'écriture, pour des apprenants du cycle primaire, semble donner lieu à des considérations confuses : pourquoi ponctuer ? Qu'est-ce que ponctuer? Certains signes plus faciles à apprendre que d'autres sont vite appropriés par les apprenants (le point d'interrogation pour poser une question), d'autres ne le sont pas. "Ces systèmes font l'objet chez l'enfant d'une lente acquisition en mémoire, qui prend place parallèlement à l'acquisition des fonctions de ces marques dans le texte » (Favart, 2005, p. 309).

L'intérêt de cette étude est de décrire et de comprendre quelques indices (ceux des programmes, des manuels scolaires et des cahiers d'activités) qui peuvent illustrer la façon dont est perçu l'acte de ponctuer par rapport à l'acte d'écrire. Selon nous, dès ses premiers contacts avec l'écrit, le jeune apprenant doit acquérir des compétences aussi bien pour la compréhension et la maîtrise du code alphabétique de la langue française, que du code graphique. Les deux systèmes ne peuvent fonctionner qu'en complémentarité. « $N u l$ aujourd'hui ne songerait sérieusement à remettre en cause l'importance de la ponctuation, tant sur le plan de l'aide à la lecture et à l'expression, que de la clarté des idées et de l'écriture au sens large du terme » (Catach, 1996, p. 3).

Comment les textes officiels prescrivent-ils l'acquisition de la ponctuation au primaire, pour les apprenants de la $3^{\text {ème }}$ année primaire (8-9ans), la $4^{\text {ème }}$ année primaire (9-10 ans) et la $5^{\text {ème }}$ année primaire (10-11 ans) ? Comment s'articule ce genre d'apprentissage pour ces trois niveaux? Y a-t-il une stratégie de progression pour l'objet à enseigner ? Que peut-on observer dans les manuels scolaires de la langue française comme contenus en rapport avec la ponctuation? Comment un tel savoir est-il présenté? De quelle manière a-t-on élaboré les exercices en relation avec l'application des programmes et les compétences visées, dans les cahiers d'activités? Notre approche est descriptive et analytique, elle permet à la fois d'observer, de recueillir des données et d'analyser les contenus des programmes et des manuels scolaires en matière de ponctuation. Selon nous, l'élaboration des programmes doit mesurer la complexité et l'utilité de l'apprentissage de la ponctuation. Une répartition d'un tel 
apprentissage dans les trois niveaux doit être raisonnée, tout en prenant en considération les apprentissages à poursuivre dans les cycles supérieurs (collège et lycée). Une analyse d'un corpus d'une cinquantaine d'apprenants appartenant à deux écoles différentes, nous a parue utile, afin d'examiner la recevabilité des activités (présentation : consignes) et afin de vérifier la compatibilité entre les compétences visées et les programmes.

\section{État des lieux et méthodologie}

Les réflexions qui suivent procèdent de l'observation des programmes institutionnels, des contenus bien spécifiques des manuels scolaires du cycle primaire, ainsi que les activités proposées dans les cahiers d'activité des apprenants pour chaque niveau : une façon de rechercher, comment ces derniers abordent la transposition didactique, d'un savoir savant à un savoir scolaire en matière de ponctuation.

\section{Prescriptions ministérielles pour l'apprentissage de la ponctuation}

Dans le respect des finalités de l'éducation définies dans le Chapitre I, Article 2 de la Loi d'orientation sur l'éducation nationale ${ }^{1}$, l'école algérienne a pour mission de:

- assurer aux élèves l'acquisition de connaissances dans les différents champs disciplinaires et la maîtrise des outils intellectuels et méthodologiques de la connaissance facilitant les apprentissages et préparant à la vie active.

- doter les élèves de compétences pertinentes, solides et durables susceptibles d'être exploitées à bon escient dans des situations authentiques de communication et de résolution de problèmes et qui les rendent aptes à apprendre toute leur vie, à prendre une part active dans la vie sociale, culturelle et économique et à s'adapter aux changements.

C'est dans cette perspective, que nous proposons de jeter de la lumière sur ce qui a été édicté par les textes officiels par rapport à l'apprentissage de la ponctuation dans le français langue étrangère. Le programme de la $3^{\text {ème }}$ année primaire ${ }^{2}$ ne présente aucune forme théorique pour l'enseignement/apprentissage de la ponctuation, cependant, à l'écrit, il vise les compétences relatives à la ponctuation comme suit:

- utiliser une ponctuation simple(le point, le point d'interrogation, le point d'exclamation et la virgule).

Le même programme attend du jeune apprenant de langue française de:

- reconnaitre les interlocuteurs dans un dialogue. 
Nous nous interrogeons, comment peut-on reconnaitre les interlocuteurs dans un dialogue, si on n'apprend pas aux apprenants que les paroles d'un dialogue doivent être insérées entre guillemets $\langle\ll »\rangle$, et que les guillemets doivent être précédés par un deux-points $\langle$ : $>$ pour annoncer l'ouverture du dialogue. Plus encore, un dialogue suppose un échange de paroles et d'interlocuteurs. Le tour de rôle nécessite, ainsi, la présence des tirets <-> pour une meilleure présentation et une meilleure compréhension du contexte de communication (qui parle ?). Aussi, nous nous interrogeons sur la prescription suivante : l'apprentissage doit se faire au double plan de l'appropriation du signe et du sens. Changer la place d'une virgule ne changet-il pas le sens; mettre un point d'exclamation à la place d'un point d'interrogation ne dénature-t-il pas la modalité de la phrase? "Il suffit du déplacement d'une virgule pour dénaturer le sens de ma pensée. Jules Michelet» (Demanuelli, 1987, p. 44).

A ce constat, l'appropriation du signe devrait impliquer, explicitement, les signes typographiques. Le programme de la $4^{\text {ème }}$ année $^{3}$ stipule un renforcement des compétences acquises par l'apprenant de 9 à 10 ans et ce dans la continuité du programme de la $3^{\text {ème }}$ année qui constitue le socle des apprentissages premiers. Pourquoi continuer, alors, à viser les mêmes compétences de la $3^{\text {ème }}$ année, c'est-à-dire, utiliser le point délimitant à bon escient, reconnaître une forme interrogative et exclamative s'il s'agit vraiment d'une continuité ? L'un des objectifs fondamentaux de l'apprentissage de la langue française en $4{ }^{\text {ème }}$ année, c'est de produire de courtes phrases en utilisant la ponctuation appropriée. Est-ce une ponctuation textuelle ? Ou, phrastique, dont l'apprenant n'a reçu aucun enseignement dans ce sens, ni de quelle forme, ni de quelle manière (emploi de la virgule), bien que les textes officiels précisent deux sortes de composantes de compétences, en grammaire. L'une d'elles est relative à la grammaire textuelle. À ce titre, l'apprenant sera capable de:

- transformer une phrase déclarative en phrase interrogative et exclamative;

- distinguer les types et les formes de phrase dans un texte;

- ponctuer un texte court (le point, la virgule, le point d'exclamation, le point d'interrogation, le tiret);

Concernant la grammaire de phrase, l'apprenant sera capable de:

- analyser la structure élémentaire de la phrase(les différents constituants) et la relation syntaxique(les accords simples).

Nous pensons qu'à ce niveau, nous pouvons établir une similitude entre un découpage de la structure élémentaire de la phrase (sujet-verbe-complément), et un découpage en signe de ponctuation, notamment l'emploi de la virgule, en mettant l'accent sur quelques usages fautifs 
de la virgule : une virgule ne se met jamais entre un sujet et son verbe, ou entre un verbe et son complément. Si le programme de la $5^{\text {ème }}$ année ${ }^{4}$ stipule qu'à ce niveau, la langue devient un objet d'étude, un objet sans cesse en construction sur le plan textuel puis sur le plan phrastique, nous nous interrogeons, en revanche, pourquoi cibler, uniquement, les signes de ponctuation qui assurent une fonction dialogique dans le texte (les parenthèses, les tirets, les guillemets et les deux points).

\section{Les signes et les fonctions de la ponctuation, cités dans les manuels scolaires}

Le manuel scolaire constitue un outil pédagogique incontournable dans l'apprentissage, l'apprenant se réfère aussi bien à son livre qu'à son enseignant. Notre examen du manuel scolaire de $3^{\text {ème }}$ année primaire (Mina, Anissa et Sadjia, 2012) nous a dévoilé une réalité inattendue. Bien que l'apprentissage soit centré sur l'acte de parole, le terme de ponctuation ne figure nulle part dans le livre, à l'exception de la page 125. Ceci nous donne une indication sur la place que revêt la ponctuation dans les pratiques didactiques. A la page 88 du même manuel, nous avons rencontré quelque chose qui fait allusion à la ponctuation dont l'intitulé de la leçon (interroger/répondre - donner un ordre), et les exemples présentés ci-après montrent, la présence d'un point, d'un point d'interrogation et d'un point d'exclamation (s'agit-il d'un apprentissage implicite ?). Le point d'exclamation $<$ ! $>$ mis après le mot personne n'est pas pertinent. Le maître qui va justifier l'emploi de celui-ci en le mettant en relation avec sa fonction (un point d'exclamation sert à exprimer une émotion forte : la joie, l'étonnement, la peur...) ne trouvera pas quoi dire pour ce cas. Dans l'énoncé en question, l'énonciateur semble neutre en complétant sa réponse par une affirmation:

-Qui est dans la salle bain?

-Personne! Manil est dans le jardin.

Les signes de ponctuation ne sont pas des accessoires, ce sont des éléments dotés du sens, un mauvais usage de ces signes est qualifié d'erreur à dominante idéogrammique (Catach, 1980). D'où la sensibilité d'être précis dans le choix des signes de ponctuations ou ponctèmes (Catach, 1996 ; Gonac'h, Delabarre \& Lenfant, 2014).

Contrairement, à celui de la $3^{\text {ème }}$ année, le manuel scolaire de la $4^{\text {ème }}$ année (Hamida, Aîcha \& M'Hamed, 2013, p. 124-125) présente clairement une leçon de ponctuation aux pages 124 et 125 . La leçon donnée à la page 124, s'intitule : je repère la ponctuation dans les comptines, dans les poèmes. Elle s'annonce par des consignes, il est demandé au jeune apprenant de lire le poème Le rayon de lune, de déduire pourquoi il a fait des pauses et à conclure que les signes présentés ne se ressemblent pas. Il n'est pas des prérogatives de cette 
recherche de discuter la méthodologie d'enseignement appliquée pour cet apprentissage, néanmoins, nous nous arrêtons sur le contenu du cadran jaune en fin de la leçon intitulée je repère la ponctuation dans les comptines, dans les poèmes à la page 124, qui semble être un retiens de la leçon: pour découper un texte en phrase ou un poème en vers, il existe des signes de ponctuation :

- Le point(.) indique que la phrase est terminée.

- Le point d'interrogation( ?)aide à poser des questions.

- Le point d'exclamation(!)montre la joie, la colère, la peur...

- La virgule(,) sépare des mots ou des phrases.

Ces signes de ponctuation aident les lecteurs à marquer des pauses.

Le contenu d'une règle ou d'un retiens a une grande influence sur les connaissances des apprenants, essentiellement, dans leurs débuts d'acquisition d'une langue étrangère qui va rester ancré dans leurs mémoires. Chose pour laquelle, donner des informations complètes et précises, même à ce niveau, s'avère d'une importance cruciale. Dans la mesure où l'on cerne la fonction de la ponctuation dans le fait de marquer des pauses pour les lecteurs. Cela ne vat-il pas enraciner une représentation chez l'apprenant, d'un rôle typiquement facultatif de la ponctuation, une fonction similaire à faire des arrêts pour respirer. Qu'en est-il des scripteurs? Et de construction de sens?

Aussi, une mise à jour, quant à la présentation des signes de ponctuation dans le manuel, tel que l'on constate en page 124, est nécessaire; désormais, les signes de ponctuation ne devront pas être présentés entre guillemets, de la façon «? », puisque ces derniers font eux-mêmes partie de la ponctuation. Les grammairiens et les linguistes les présentent ainsi $<$ ?> pour éloigner toute confusion. Toutefois, nous relevons une distance entre ce que stipulent les textes officiels en traçant les objectifs suivants:

- produire de courtes phrases en utilisant la ponctuation appropriée;

- compléter des dialogues par une ou deux répliques;

- produire de courts textes (quelques phrases) pour dialoguer, raconter et/ou décrire.

Et les activités qui se présentent dans la page 125, dont le travail demandé aux apprenants, consiste à mettre des points d'interrogations et des points d'exclamation. Il s'agit de compétences déjà acquises en $3^{\text {ème }}$ année. L'examen du manuel scolaire de la cinquième année (Lamine, Sacia, Nafissa, Fouzia \& Mohammed, 2011), a enfin confirmé nos doutes sur l'état de l'apprentissage de la ponctuation, au primaire : il se fait, d'une manière répétitive (un même enseignement dans les trois niveaux) au lieu d'un enseignement progressif et complémentaire d'un niveau à un autre. Et par conséquent, un apprenant de la cinquième 
année (la troisième année d'apprentissage du français langue étrangère) acquit une compétence démarcative qui sert à délimiter une phrase en fonction de la modalité de celle-ci. Le retiens de la page 15 du même manuel, bien qu'il ait cité explicitement une fonction pertinente de la ponctuation qu'est la compréhension, en garde cependant les mêmes connaissances données en $3^{\text {ème }}$ année et en $4^{\text {ème }}$ année. Plus encore, une définition a donné l'impression de revenir en arrière : une phrase commence par une majuscule et se termine par un point, cette phrase est considérée, dans toutes les méthodologies d'enseignement/ apprentissage, comme le point de départ des cours sur la ponctuation, elle ne devrait pas avoir sa place à ce niveau (celui de la $5^{\text {ème }}$ année). Une autre connaissance semble être donnée pour enrichir ou ajouter un plus par rapport à ce qui a été donné à des niveaux inférieurs. Il existe aussi d'autres signes de ponctuation:

- les virgules (,) pour séparer les mots;

- les guillemets $(<<>>)$ et les tirets $(-)$ pour les dialogues.

Ces informations ne s'avèrent guère nouvelles ni complémentaires, puisqu' qu'elles ont fait l'objet des activités données aux apprenants de la ${ }^{\text {ème }}$ année (analysées ci- dessous). Ailleurs, pour ce niveau, des travaux de recherches s'inquiètent sur la ponctuation du mot en relevant les constats suivants : "le fait de ne pas renforcer les signes de ponctuation jugés d'usage fréquent avec d'autres signes tels que l'apostrophe $\left\langle\right.$ '>, le trait d'union $<->_{-}$et l'espace $<\phi>$ qui est jugé absent de la grammaire scolaire et de la grammaire traditionnelle» (Chabanne, 1998, p. 226).

\section{Composantes des compétences et activités d'entrainement}

A titre d'illustration, nous avons choisi de mener une recherche qui met l'accent sur les activités d'entraînement, faisant partie du processus d'apprentissage de la ponctuation. Pour ce faire, nous avons opté pour le choix des activités prises du cahier d'activités de la $4^{\text {ème }}$ année. A notre sens, il s'agit d'activités variées, allant du simple repérage des signes jusqu'à l'adaptation des signes à un dialogue. Nous ne nous sommes pas intéressés aux compétences démarcatives associées aux compétences scripturales, notre objectif était d'examiner le processus d'apprentissage de la ponctuation en le mesurant avec sa conception et son élaboration ; pour cela nous avons éloigné toute surcharge cognitive à laquelle les apprenants seraient confrontés dans leurs productions écrites. La quête d'un transcodage phonie/graphie dans une langue qui vient de naître, peut rendre l'évaluation des compétences en ponctuation non exacte, dans la mesure où le code alphabétique, pour les jeunes apprenants, prime sur le code typographique (jugé secondaire). Les activités de la page 30 du cahier d'activités de la 
$4^{\text {ème }}$ année présente quatre activités, dont la composante de la compétence visée, s'inscrit dans : Assurer la présentation d'un écrit comme le stipule le programme de la $4^{\text {ème }}$.

\section{Présentation des activités}

La première activité avait pour consigne d'entourer en rouge les signes de ponctuation dans le texte. Il s'agit d'un micro-texte de trois lignes et demie qui contient 47 mots et un effectif de signes de ponctuation de 11 emplacements. Les signes que comporte le texte sont : \{,/./?/!/: I...\}.

La deuxième activité comporte deux consignes : placer les points et entourer la lettre qui doit être en majuscule. Une information était donnée entre parenthèses (il y a 5 phrases) afin de faciliter la tâche aux jeunes apprenants : il s'agit d'une évaluation semi guidée. Le micro-texte de trois lignes et demie comporte 54 mots et un effectif de signes de ponctuation de trois $\{, /: /$.$\} .$

La troisième activité comporte deux consignes : mets les signes de ponctuation (.)- (?)(!) qui conviennent dans le texte à la place de ( ), et entoure les lettres qui doivent être en majuscule. Le micro-texte de deux lignes et demie comporte 42 mots avec quatre virgules et six emplacements ( ) de signes de ponctuation que l'apprenant devrait compléter.

La quatrième activité concerne la réécriture d'un dialogue et le remplacement des barres par les signes de ponctuation qui manquent. Cette fois-ci aucun signe n'a été donné.

\section{Cadre d'analyse}

Notre corpus d'étude a concerné un groupe de cinquante-deux apprenants de la $4^{\text {ème }}$ année (9 à 10 ans), appartenant à deux écoles primaires dans la wilaya de Sidi Bel Abbes ; à savoir TALEB Salima et AFFEN Fatima Zohra (jugées parmi les écoles ayant souvent un classement satisfaisant durant les épreuves de la sixième année). Ce corpus est constitué sur la base des exercices relevés dans les cahiers d'activités des apprenants concernant la ponctuation. Nous avons disposé les 25 apprenants $\left(\begin{array}{llll}9 & \text { à } & 10 & \text { ans }\end{array}\right)$ appartenant à l'école «TALEB Salima» dans une salle de classe, et les 27 apprenants (9 à 10ans) appartenant à l'école «AFFEN Fatima » dans une autre salle. Nous avons remis à chaque élève une copie des exercices, nous avons demandé aux élèves de bien lire la consigne et de faire les quatre activités dans un temps de $20 \mathrm{mn}$. Le choix des 4 exercices n'était pas aléatoire, d'une part, ce sont des exercices d'application de ce qui est censé donné durant le cours (à propos de la ponctuation), et d'autre part, nous avons ciblé la diversification des objectifs (repérage des signes de ponctuation-emplacement des signes-le choix du signe qu'il 
faut à la place qu'il faut-la mise en forme d'un dialogue). Les 52 copies ont été examinées. Nous les avons, ensuite, analysées selon des critères relatifs à notre thème de recherche, à savoir : la formulation des consignes, la pertinence dans le choix des activités, la réceptivité des apprenants, c'est -à-dire leurs comportements observables vis-à-vis du travail demandé.

\section{Résultats et analyses}

La première activité avait pour objectifs de tester des compétences de repérage et d'identification des signes de ponctuation préexistant dans le texte. Les résultats obtenus montrent que la quasi-totalité des apprenants ont réalisé cette activité à l'exception de deux apprenants. Quelques omissions ont été relevées, notamment, en ce qui concerne les trois points de suspension, les deux points et les virgules (précisément la virgule qui se place après le complément de phrase hier). Les compétences de détection semblent largement être acquises. Quant à la deuxième activité, basée nettement sur la ponctuation textuelle (la segmentation du texte en cinq phrases), elle avait comme attentes de tester les compétences de délimitation des phrases (majuscule/point). Les performances observées ne semblent pas rassurantes. Les apprenants semblent avoir des difficultés au niveau de la compréhension de la consigne : $25 \%$ d'apprenants ont utilisé des virgules, des points d'interrogation et des points d'exclamation alors que seul l'usage du point leur a été demandé. 35\% d'apprenants ont utilisé le point d'une façon non raisonnée, c'est-à-dire que le point n'est pas utilisé pour clore une phrase. Par exemple :

[...] d'aller. chez la paysanne

[...] de sa drôle. de barbe

$[\ldots]$ va rendre visite à un vieux. Sage du village.

Nous pensons que les activités d'applications, données dans les cahiers d'activités des apprenants de la quatrième année ayant pour objectif la vérification des compétences visées par l'unique leçon sur la ponctuation (Hamida, et al., 2013, p. 124) ne sont pas en adéquation avec le contenu de la leçon en question. Selon nous, ces activités devraient être précédées par d'autres apprentissages qui devraient se faire antérieurement tels que: qu'est-ce qu'une phrase ? Comment associer la définition de la ponctuation à la description de ses composantes et de leurs fonctionnements? " Traiter exhaustivement cette question pour proposer un programme d'apprentissage cohérent, articulant savamment les différentes notions, catégories, fonctions impliquées» (David \& Vaudrey-Luig, 2014, p.6). Seuls, 20\% des apprenants ont réussi le bon usage des points et des majuscules; en revanche, certains apprenants semblent confondre entre la majuscule de la phrase et la majuscule du nom propre 
Barbe-de-plumes cité trois fois dans le texte ${ }^{5}$. La troisième activité a pour but de tester les compétences des apprenants par rapport à la substitution du point déclaratif par le point interrogatif ou exclamatif. L'analyse du corpus a abouti aux constats suivants:

- une performance de $90 \%$ quant à l'usage du point d'exclamation après l'interjection oh!

- une performance de plus de $80 \%$ quant à l'usage du point d'interrogation après la question qu'est-ce qu'il y a dedans.

En ce qui concerne l'expression que ce cartable est lourd, $60 \%$ des apprenants ont mis un point d'interrogation, $20 \%$ ont mis un point et $20 \%$ ont mis un point d'exclamation. Nous supposons qu'il y a eu confusion entrequece et qu'est-ce que pour la majorité des apprenants. Quant à l'expression des briques, pour répondre à la question qu'est-ce qu'il y a dedans?, nous avons relevé trois types de réponses:

- $10 \%$ seulement, d'apprenants ont mis un point d'exclamation : des briques !

- $30 \%$ ont mis un point d'interrogation : des briques ?

- $60 \%$ ont mis un point : des briques.

Cette activité nous a amenés à nous poser les questions suivantes: comment évaluer ? Quels sont les critères d'évaluation? Comment concevoir des activités qui permettent une évaluation stricte des compétences acquises? Qu'est ce qui est pertinent dans ce genre d'évaluation? Les résultats obtenus dans la quatrième activité, ont permis de relever des constats qui nous ont révélé d'autres questionnements par rapport à l'exercice donné ci-après:

- Bonjour // qui es-tu // dit le petit prince //

- Bonjour // je suis un renard et toi // qui es-tu //

- Je suis le petit prince// je recherche les hommes //

Bien que $80 \%$ aient réussi à mettre un point après l'expression dit le petit prince, $20 \%$ ont mis un deux-points <:>. Ce constat ne nous a pas laissé indifférents, nous nous sommes interrogés, qui est plus compétent? Les apprenants qui ont mis un point? Ou les $20 \%$ qui ont mis un deux-points? Qui ont, peut-être, compris qu'il s'agit d'un dialogue et qui avaient, peut-être, une représentation du genre le petit prince dit. Nous nous demandons si les marques $\mathrm{du}$ dialogue faisaient partie des critères de réussite préétablis, et dans ce cas, les emplacements prévus pour les signes de ponctuation attendus de l'apprenant dans cette activité, sont erronés. Qu'en est-il des emplacements des tirets et des guillemets ? Si ce n'était pas le cas, pourquoi parler de dialogue ? S'agit-il alors d'une simplification de notions qu'on croit adapter aux capacités des apprenants, afin d'éviter la surcharge cognitive : «L'absence 
de signe de ponctuation du dialogue n'est pas le reflet d'une méconnaissance du code mais plutôt la conséquence d'une surcharge cognitive» (Guénez, 2014, p. 72).

D'autres activités, à notre sens, peuvent donner un impact très positif sur l'usage et l'importance de la ponctuation dans la compréhension. Exemples:

« Vous n'aurez pas vos cadeaux comme prévu. »

« Vous n'aurez pas vos cadeaux, comme prévu. »

Amine dit : «Imad est méchant ».

Amine, dit Imad, est méchant.

Les jeunes apprenants peuvent, d'une manière ludique, prendre conscience de la gravité que peut engendrer un usage fautif des signes de ponctuation. Pour les exemples cités ci-dessus, changer l'emplacement d'un signe ou utiliser un signe à la place d'un autre peut donner deux jugements différents.

\section{Conclusion}

A travers, l'étude descriptive et compréhensive, que nous avons menée, nous avons éclairé une réalité qui nous a parue ambigüe quant à l'apprentissage de la ponctuation. Nous pouvons affirmer, qu'il s'agit d'un écart qui existe entre les attentes des institutions et les contenus pédagogiques qui régissent les pratiques didactiques qui peuvent influencer les comportements scripturaux observables des apprenants. La nécessité de remettre en cause la stratégie d'apprentissage actuelle s'impose. Se limiter, au primaire, à un inventaire restreint de signes de ponctuation n'est pas simplifié, il est plutôt lacunaire. Le jeune apprenant de 8 à 11 ans doit prendre conscience, dès ses premiers pas dans l'apprentissage du français langue étrangère, des signes graphiques, dont Claude Tournier dit qu'ils sont Comparables au mot, pour mettre en évidence leur importance (Tournier, 1980, p. 36). Une démarche déductive (j'apprends puis j'applique) telle qu'elle était conçue dans l'ancien programme et dans les anciens livres de grammaire s'impose, notamment en troisième année (l'apprenant doit se mettre face à une leçon de ponctuation complète, où la quasi-totalité des signes de ponctuation lui seraient présentés). A partir de la quatrième année, et durant tout son cursus d'apprentissage, il aurait à faire à l'approche par compétences (je découvre, j'apprends, je m'exerce) à condition qu'il soit intégré dans des apprentissages progressifs et complémentaires. L'idée de simplification a fini par être critiquée par de nombreux chercheurs: Chabannes (1998), Paolacci (2010), Paolacci et Favart (2010). Le modèle scolaire doit prendre en considération qu'il s'agit d'un long apprentissage à entreprendre, à savoir la ponctuation du texte, de la phrase et du mot. Au plan pédagogique, on doit mesurer ce que les 
apprenants du cycle primaire auront comme apprentissage en matière de ponctuation en troisième année, en quatrième année et en cinquième année.

\section{Références bibliographiques}

Catach, N. (1980). L'orthographe française. Paris: Nathan Université.

Catach, N. (1996). La ponctuation (Histoire et système). Paris: Presses Universitaires de France Que sais-je?

Chabanne, J.C. (1998). La ponctuation dans les manuels à l'école primaire (8-10 ans) : aspects théoriques et didactiques. In J.M. Defays, M. Rosier \& F. Tilkin (Eds), A qui appartient la ponctuation? (pp. 223-241). Paris, Bruxelles: Editions Duculot.

David, J., \& Vaudrey-Luigi, S. (2014). Enseigner la ponctuation. Le français aujourd'hui, 187(3-6). DOI : 10.3917/lfa.187.0003.

Demanuelli, C. (1987). Points de repère: approche interlinguistique de la ponctuation français-anglais. Saint-Etienne: Université de Saint-Etienne.

Favart, M. (2005). Les marques de cohésion: leur rôle fonctionnel dans l'acquisition de la production écrite de texte. Psychologie française, 50: 305-322. Doi.org/10.1016/j.psfr.2005.05.006.

Gonac'h, J., Delabarre, E., \& Lenfant., M., (2014). Des signes de ponctuation en relief et en sourdine chez des élèves de CM1 normands, le français aujourd'hui, 187: 57-65. Doi.org/10.3917/1fa.187.0057.

Guénez, C, V., (2014). A la recherche de styles de scription, usages de ponctuation : erreurs, normes, créativités. Le français aujourd'hui, 187 : 67-77. Doi.org/10.3917/1fa.187.0067.

Hamida, K., Aicha, D., \& M'hamed, I. (2013). Mon livre de français. Alger: Office National des Publications.

Lamine, S., Sacia. F., Nafissa. A., Fouzia. N., \& Mohamed. N. (2011). Mon livre de français. Alger: Office National des Publications Scolaires.

Mina, M., Anissa., B., \& Sadjia., M. (2012). Mon premier livre de français. Alger: Office National des Publications Scolaires.

Paolacci, V. (2010). Les manuels scolaires de grammaire, entre savoirs linguistiques de référence et prescriptions institutionnelles. Le cas de la ponctuation dans les manuels de CM et sixième. In Actes du colloque (CDROM). Enseigner la grammaire en Francophonie : curricula, pratiques observées, formation des enseignants. Toulouse. Février.

Paolacci, V., \& Favart, M. (2010). Traitement des marques de cohésion par les jeunes scripteurs : l'utilisation de la ponctuation et des connecteurs à l'entrée en sixième. Approche linguistique, cognitive et didactique. Langages, 177: 117-132. Doi.org/10.3917/lang.177.0113.

Tournier, C. (1980). L'histoire des idées sur la ponctuation; des débuts de l'imprimerie à nos jours. Langue française, 45: 28-41. Doi.org/10.3406/1fr.1980.5261

\footnotetext{
${ }^{1}$ Loi $\mathrm{n}^{\circ} 08-04$ du 23 janvier 2008 portant loi d'orientation sur l'éducation nationale. Journal officiel de la république algérienne $n^{\circ} 4$.

${ }^{2}$ Programme de français de la troisième année primaire, direction de l'enseignement fondamentale, commissions nationales des programmes, Alger, juin 2011.

${ }^{3}$ Programme de français de la quatrième année primaire, direction de l'enseignement fondamentale, commissions nationales des programmes, Alger, juin 2011.
} 
${ }^{4}$ Programme de français de la cinquième année primaire, direction de l'enseignement fondamentale, commissions nationales des programmes, Alger, juin 2011.

${ }^{5}$ Le choix du nom du personnage Barbe-de-plumes n'est pas judicieux, il risque de déconcentrer l'apprenant (pour le jeune apprenant, cela pourrait être pris comme une phrase).

\section{Remerciements}

Un grand merci à mon directeur de thèse Prof. Belabbas Missouri (Université de Sidi Bel Abbès- Algérie) et la professeure Veronique Lagae (Université de Valenciennes et du Hainaut-Cambrésis Campus du Mont Houy, Valenciennes -France) qui m’ont guidé dans mes premières tentatives de recherche sur le sujet en question et aussi d'avoir apporté les corrections nécessaires.

\section{Références de l'auteur}

Hassiba Addou est doctorante à l’Université Djillali Liabès de Sidi Bel Abbès (Algérie). Elle prépare actuellement un doctorat en didactique du français langue étrangère (FLE) portant sur le traitement didactique des compétences démarcatives: l'utilisation de la ponctuation chez les apprenants en classe de FLE. Elle occupe aussi un poste d'enseignante de français au cycle secondaire et fait partie d'une commission de certificat d'aptitude professionnelle de l'enseignement secondaire.

E-mail: addouhassiba@yahoo.fr

Pour citer cet article:

Addou, H. (2016). Analyse du matériel pédagogique en matière de ponctuation dans l'école primaire algérienne.

Bellaterra Journal of Teaching \& Learning Language \& Literature, 9(1), 71-83. DOI:

http://dx.doi.org/10.5565/rev/jt13.650 